\title{
Major Adverse Cardiac Events After Radiation Therapy in Lung Cancer
}

\author{
Ramsey Omari ${ }^{1}$, Charles Curtis ${ }^{2}$, Nichole Burkett ${ }^{3}$, Michael Weisman ${ }^{2}$, Xiaofeng Chen $^{2}$, Tim \\ Lautenschlaeger ${ }^{2}$
}

${ }^{1}$ Indiana University School of Medicine, Indianapolis, IN; ${ }^{2}$ Department of Radiation Oncology, Indiana University School of Medicine, Indianapolis, IN; ${ }^{3}$ Marian University College of

Osteopathic Medicine, Indianapolis, IN

\section{Motivation:}

Receiving radiation to the heart has been recognized as a risk factor for the development of major adverse cardiovascular events (MACEs) for many years. However, recent data suggests that radiation dosing to substructures of the heart serve as a better surrogate for evaluating the risk of developing a MACE than whole heart radiation dose. Recent papers suggest that dosing to the left anterior descending artery (LAD) can be used as a robust marker for cardiotoxicity risk; however, this association lacks corroborative data and is currently not incorporated into clinically routine care.

\section{Problem:}

In this paper we seek to investigate the relationship between radiation dose to the LAD and risk of developing a MACE in lung cancer patients treated with curative intent radiation.

\section{Approach:}

Chart review to confirm the presence of MACE events was performed in patients who were identified based on elevated troponin values to potentially have had a MACE after receiving their last dose of radiation therapy. Patients who had multiple courses of radiation therapy separated in time (>60 days) that received greater than 0.2 Gy whole heart dose during their subsequent courses before having a MACE were excluded. Selected patients were then stratified based on presence cardiovascular co-morbidities. Contours of patient's LADs were made after patient selection, and will be verified by an expert (e.g., cardiologist or thoracic radiologist).

\section{Results:}

Dose to the LAD will be calculated and an assessment of the correlation between radiation dose and risk of having a MACE will be made. Analysis will assess the cardiac event rate at various times as well as time to MACE.

\section{Implications:}

This paper can help set a quantifiable standard with which radiation oncologists can use to minimize their patient's risk of developing a MACE by minimizing radiation dosing to specific cardiac substructures while maintaining tumor coverage. 